doi: $10.13108 / 2021-13-1-131$

\title{
ON GEOMETRIC PROPERTIES OF MORREY SPACES
}

\author{
H. GUNAWAN, D.I. HAKIM, A.S. PUTRI
}

\begin{abstract}
The study of Morrey spaces is motivated by many reasons. Initially, these spaces were introduced in order to understand the regularity of solutions to elliptic partial differential equations [1. In line with this, many authors study the boundedness of various integral operators on Morrey spaces. In this article, we are interested in their geometric properties, from functional analysis point of view. We show constructively that Morrey spaces are not uniformly non- $\ell_{n}^{1}$ for any $n \geqslant 2$. This result is sharper than earlier results, which showed that Morrey spaces are not uniformly non-square and also not uniformly nonoctahedral. We also discuss the $n$-th James constant $C_{\mathrm{J}}^{(n)}(X)$ and the $n$-th Von NeumannJordan constant $C_{\mathrm{NJ}}^{(n)}(X)$ for a Banach space $X$, and obtain that both constants for any Morrey space $\mathcal{M}_{q}^{p}\left(\mathbb{R}^{d}\right)$ with $1 \leqslant p<q<\infty$ are equal to $n$.
\end{abstract}

Keywords: Morrey spaces, uniformly non- $\ell_{n}^{1}$-ness, $n$-th James constant, $n$-th Von Neumann-Jordan constant.

Mathematics Subject Classification: 46B20, 42B35

\section{INTRODUCTION}

For $1 \leqslant p \leqslant q<\infty$, the Morrey space $\mathcal{M}_{q}^{p}=\mathcal{M}_{q}^{p}\left(\mathbb{R}^{d}\right)$ is the set of all measurable functions $f$ such that

$$
\|f\|_{\mathcal{M}_{q}^{p}}:=\sup _{a \in \mathbb{R}^{d}, R>0}|B(a, R)|^{\frac{1}{q}-\frac{1}{p}}\left(\int_{B(a, R)}|f(y)|^{p} d y\right)^{\frac{1}{p}}<\infty,
$$

where $|B(a, R)|$ denotes the Lebesgue measure of the open ball $B(a, R)$ in $\mathbb{R}^{d}$, with center $a$ and radius $R$. Morrey spaces are Banach spaces (see, e.g., [12]). For $p=q$, the space $\mathcal{M}_{q}^{q}$ is identical with the space $L^{q}=L^{q}\left(\mathbb{R}^{d}\right)$, the space of $q$-th power integrable functions on $\mathbb{R}^{d}$. Knowledge of Morrey spaces is important in studying regularity of solutions to elliptic partial differential equations [1].

In [4], three geometric constants have been computed for Morrey spaces. The first two constants, namely Von Neumann-Jordan constant and James constant, are closely related to the notion of uniformly non-squareness of (the unit ball in) a Banach space [5, 6, 8]. For a general Banach space $\left(X,\|\cdot\|_{X}\right)$, the constants are defined by

$$
C_{\mathrm{NJ}}(X):=\sup \left\{\frac{\|x+y\|_{X}^{2}+\|x-y\|_{X}^{2}}{2\left(\|x\|_{X}^{2}+\|y\|_{X}^{2}\right)}: x, y \in X \backslash\{0\}\right\},
$$

and

$$
C_{\mathrm{J}}(X):=\sup \left\{\min \left\{\|x+y\|_{X},\|x-y\|_{X}\right\}: x, y \in S_{X}\right\},
$$

respectively. Here $S_{X}:=\left\{x \in X:\|x\|_{X}=1\right\}$ denotes the unit sphere in $X$. A few basic facts about these constants are:

- $1 \leqslant C_{\mathrm{NJ}}(X) \leqslant 2$ and $C_{\mathrm{NJ}}(X)=1$ if and only if $X$ is a Hilbert space [7].

- $\sqrt{2} \leqslant C_{\mathrm{J}}(X) \leqslant 2$ and $C_{\mathrm{J}}(X)=\sqrt{2}$ if $X$ is a Hilbert space [3].

H. Gunawan, D.I. Hakim, A.S. Putri, On Geometric properties of Morrey spaces.

(c) H. Gunawan, D.I. Hakim, A.S. Putri. 2021.

This work is supported by P3MI-ITB 2020 Program.

Submitted May 7, 2020. 
Note also that, for $1 \leqslant p \leqslant \infty$, we have [2, 3]:

$$
C_{\mathrm{NJ}}\left(L^{p}\right)=\max \left\{2^{\frac{2}{p}-1}, 2^{1-\frac{2}{p}}\right\} \quad \text { and } \quad C_{\mathrm{J}}\left(L^{p}\right)=\max \left\{2^{\frac{1}{p}}, 2^{1-\frac{1}{p}}\right\} .
$$

The two constants measure the non-squareness of (the unit ball in) a Banach space. The larger the constants, the lesser round the unit ball. We note from [5, 8] that a Banach space $X$ is uniformly non-square if and only if $C_{\mathrm{J}}(X)<2$ or, equivalently, $C_{\mathrm{NJ}}(X)<2$.

In this paper, the main objects of our study are Morrey spaces. We know that Morrey spaces $\mathcal{M}_{q}^{p}$ contain Lebesgue spaces $L^{q}$. While $L^{q}$ are uniformly non-square for $1<q<\infty$, we have the following result which tell us that $\mathcal{M}_{q}^{p}$ are not uniformly non-square whenever $1 \leqslant p<q<\infty$.

Theorem 1.1. ([4]). For $1 \leqslant p<q<\infty$, we have $C_{\mathrm{NJ}}\left(\mathcal{M}_{q}^{p}\right)=C_{\mathrm{J}}\left(\mathcal{M}_{q}^{p}\right)=2$.

In [11], it was shown that Morrey spaces are not uniformly non-octahedral, that is, there exists no $\delta>0$ such that

$$
\min \|f \pm g \pm h\|_{\mathcal{M}_{q}^{p}} \leqslant 3(1-\delta)
$$

for all $f, g, h \in \mathcal{M}_{q}^{p}$ with

$$
\|f\|_{\mathcal{M}_{q}^{p}}=\|g\|_{\mathcal{M}_{q}^{p}}=\|h\|_{\mathcal{M}_{q}^{p}}=1 .
$$

Here the minimum is taken over all choices of signs in the expression $f \pm g \pm h$. We recall that a Banach space $\left(X,\|\cdot\|_{X}\right)$ is uniformly non-octahedral if there exists a $\delta>0$ such that

$$
\min \|x \pm y \pm z\|_{X} \leqslant 3(1-\delta)
$$

for every $x, y, z \in S_{X}$. Precisely, we have the following theorem.

Theorem 1.2. ([11]). Let $1 \leqslant p<q<\infty$. Then, for every $\delta>0$, there exist $f, g, h \in \mathcal{M}_{q}^{p}$ depending on $\delta$ with

$$
\|f\|_{\mathcal{M}_{q}^{p}}=\|g\|_{\mathcal{M}_{q}^{p}}=\|h\|_{\mathcal{M}_{q}^{p}}=1
$$

such that

$$
\|f \pm g \pm h\|_{\mathcal{M}_{q}^{p}}>3(1-\delta)
$$

for all choices of the signs.

In this paper we show constructively that Morrey spaces are not uniformly non- $\ell_{n}^{1}$, see [5, Definition 2.1]. The result is not only more general than the previous ones, but also sharper than knowing results stating that Morrey spaces are neither uniformly non-square nor uniformly non-octahedral; if $X$ is not uniformly non- $\ell_{n}^{1}$ for $n \geqslant 3$, then $X$ is not uniformly non- $\ell_{n-1}^{1}$. In addition, given a Banach space $X$, we discuss the $n$-th Von Neumann-Jordan constant $C_{\mathrm{NJ}}^{(n)}(X)$ and the $n$-th James constant $C_{\mathrm{J}}^{(n)}(X)$ for $n \geqslant 2$. These two constants were studied in [9] and [10], respectively. We show that for each Morrey space $\mathcal{M}_{q}^{p}$ with $1 \leqslant p<q<\infty$ both constants are equal to $n$. We also indicate that Morrey spaces are not uniformly $n$-convex for $n \geqslant 2$.

\section{2. $\mathcal{M}_{q}^{p}$ ARE NOT UNIFORMLY NON- $\ell_{n}^{1}$}

Before we present our main theorems, we prove several lemmata. Unless otherwise stated, we assume that $1 \leqslant p<q<\infty$.

Lemma 2.1. Let $f(x):=|x|^{-d / q}$. Then $f \in \mathcal{M}_{q}^{p}$ with

$$
\|f\|_{\mathcal{M}_{q}^{p}}=\left(\frac{\omega_{d-1}}{d}\right)^{\frac{1}{q}}\left(\frac{q}{q-p}\right)^{\frac{1}{p}}
$$

where $\omega_{d-1}$ denotes the 'area' of the unit sphere in $\mathbb{R}^{d}$. 
Proof. For each $r>0$, one may compute that

$$
|B(0, r)|^{\frac{1}{q}-\frac{1}{p}}\left(\int_{B(0, r)}|x|^{-d p / q} d x\right)^{\frac{1}{p}}=\left(\frac{\omega_{d-1}}{d}\right)^{\frac{1}{q}}\left(\frac{q}{q-p}\right)^{\frac{1}{p}}
$$

which is independent of $r$. Since the integral of $f$ over $B(a, r)$ is less than that over $B(0, r)$ for each $a \in \mathbb{R}^{d}$, we conclude that

as claimed.

$$
\|f\|_{\mathcal{M}_{q}^{p}}=\left(\frac{\omega_{d-1}}{d}\right)^{\frac{1}{q}}\left(\frac{q}{q-p}\right)^{\frac{1}{p}}
$$

Lemma 2.2. Let $f(x):=|x|^{-d / q}$ and $R>1$. Then, for any $c_{1}, c_{2}>0$, we have

$$
\left|B_{c_{1} R}\right|^{1 / q-1 / p}\left(\int_{\left\{x: c_{1}<|x|<c_{1} R\right\}}|f(x)|^{p} d x\right)^{\frac{1}{p}}=\left|B_{c_{2} R}\right|^{1 / q-1 / p}\left(\int_{\left\{x: c_{2}<|x|<c_{2} R\right\}}|f(x)|^{p} d x\right)^{\frac{1}{p}},
$$

where $B_{c_{1} R}$ and $B_{c_{2} R}$ are balls centered at the origin with radii $c_{1} R$ and $c_{2} R$.

Proof. It suffices to prove that (2.1) holds for arbitrary $c_{1}>0$ and $c_{2}=1$. But this is immediate by the change of variable $x=c_{1} x^{\prime}$.

As a consequence of the above lemma, we have the following corollary, which is an important ingredient in the proof of our main theorems.

Corollary 2.1. Let $f(x):=|x|^{-\frac{d}{q}}$. For $\varepsilon \in(0,1)$ and $k \in \mathbb{Z}_{+}$, we denote

$$
f_{\varepsilon, k}:=f \chi_{\left\{x: \varepsilon^{k+1}<|x|<\varepsilon^{k}\right\}} \text {. }
$$

Then $f_{\varepsilon, k} \in \mathcal{M}_{q}^{p}$ with

$$
\left\|f_{\varepsilon, k}\right\|_{\mathcal{M}_{q}^{p}} \geqslant\left(1-\varepsilon^{d-\frac{d p}{q}}\right)^{\frac{1}{p}}\|f\|_{\mathcal{M}_{q}^{p}}
$$

Proof. In view of Lemma 2.2, it suffices to prove that

$$
\left\|f_{\varepsilon, 0}\right\|_{\mathcal{M}_{q}^{p}} \geqslant\left(1-\varepsilon^{d-\frac{d p}{q}}\right)^{\frac{1}{p}}\|f\|_{\mathcal{M}_{q}^{p}}
$$

We observe that

$$
\left\|f_{\varepsilon, 0}\right\|_{\mathcal{M}_{q}^{p}} \geqslant|B(0,1)|^{\frac{1}{q}-\frac{1}{p}}\left(\int_{\{x: \varepsilon<|x|<1\}}|f(x)|^{p} d x\right)^{\frac{1}{p}}=\left(\frac{\omega_{d-1}}{d}\right)^{\frac{1}{q}}\left(\frac{q}{q-p}\right)^{\frac{1}{p}}\left(1-\varepsilon^{d-\frac{d p}{q}}\right)^{\frac{1}{p}} .
$$

Hence, by Lemma 2.1, the desired inequality follows.

We are now ready to state our main results. Our first theorem is the following.

Theorem 2.1. For $1 \leqslant p<q<\infty$, the Morrey space $\mathcal{M}_{q}^{p}$ is not uniformly non- $\ell_{n}^{1}$ for any $n \geqslant 2$, that is, for each $\delta \in(0,1)$, there exist $F_{1}, F_{2}, \ldots, F_{n} \in \mathcal{M}_{q}^{p}$ depending on $\delta$ with $\left\|F_{i}\right\|_{\mathcal{M}_{q}^{p}}=1$ for $i=1,2, \ldots, n$, such that

$$
\left\|F_{1} \pm F_{2} \pm \cdots \pm F_{n}\right\|_{\mathcal{M}_{q}^{p}}>n(1-\delta)
$$

for all choices of signs.

Proof. To understand the idea of the proof, let us first illustrate how the proof goes for $n=3$. Given $\delta \in(0,1)$, we choose $\varepsilon \in\left(0,\left(1-(1-\delta)^{p}\right)^{\frac{q}{d q-d p}}\right)$. For $f(x):=|x|^{-\frac{d}{q}}$ and $k \in \mathbb{Z}_{+}$, put $f_{\varepsilon, k}:=f \chi_{\left\{x: \varepsilon^{k+1}<|x|<\varepsilon^{k}\right\}}$. Now write

$$
\begin{aligned}
& f_{1}:=(+1,+1,+1,+1):=f_{\varepsilon, 3}+f_{\varepsilon, 2}+f_{\varepsilon, 1}+f_{\varepsilon, 0}, \\
& f_{2}:=(+1,+1,-1,-1):=f_{\varepsilon, 3}+f_{\varepsilon, 2}-f_{\varepsilon, 1}-f_{\varepsilon, 0}, \\
& f_{3}:=(+1,-1,+1,-1):=f_{\varepsilon, 3}-f_{\varepsilon, 2}+f_{\varepsilon, 1}-f_{\varepsilon, 0} .
\end{aligned}
$$


Observe that $\left\|f_{i}\right\|_{\mathcal{M}_{q}^{p}}=\left\|f \chi_{\left\{x: \varepsilon^{4}<|x|<1\right\}}\right\|_{\mathcal{M}_{q}^{p}}$ for $i=1,2,3$, and that

$$
\begin{aligned}
& 3 f_{\varepsilon, 3} \leqslant\left|f_{1}+f_{2}+f_{3}\right| \leqslant 3 f \chi_{\left\{x: \varepsilon^{4}<|x|<1\right\}}, \\
& 3 f_{\varepsilon, 2} \leqslant\left|f_{1}+f_{2}-f_{3}\right| \leqslant 3 f \chi_{\left\{x: \varepsilon^{4}<|x|<1\right\}}, \\
& 3 f_{\varepsilon, 1} \leqslant\left|f_{1}-f_{2}+f_{3}\right| \leqslant 3 f \chi_{\left\{x: \varepsilon^{4}<|x|<1\right\}}, \\
& 3 f_{\varepsilon, 0} \leqslant\left|f_{1}-f_{2}-f_{3}\right| \leqslant 3 f \chi_{\left\{x: \varepsilon^{4}<|x|<1\right\}} .
\end{aligned}
$$

By virtue of Corollary 2.1, we have

$$
3\left(1-\varepsilon^{d-\frac{d p}{q}}\right)^{\frac{1}{p}}\|f\|_{\mathcal{M}_{q}^{p}} \leqslant\left\|f_{1} \pm f_{2} \pm f_{3}\right\|_{\mathcal{M}_{q}^{p}} \leqslant 3\left\|f \chi_{\left\{x: \varepsilon^{4}<|x|<1\right\}}\right\|_{\mathcal{M}_{q}^{p}}
$$

for all choices of signs. For $i=1,2,3$, define

$$
F_{i}:=\frac{f_{i}}{\left\|f_{i}\right\|_{\mathcal{M}_{q}^{p}}}
$$

Then, $\left\|F_{i}\right\|_{\mathcal{M}_{q}^{p}}=1$ for $i=1,2,3$, and

$$
\left\|F_{1} \pm F_{2} \pm F_{3}\right\|_{\mathcal{M}_{q}^{p}}=\frac{\left\|f_{1} \pm f_{2} \pm f_{3}\right\|_{\mathcal{M}_{q}^{p}}}{\left\|f \chi_{\left\{x: \varepsilon^{4}<|x|<1\right\}}\right\|_{\mathcal{M}_{q}^{p}}} \geqslant \frac{3\left(1-\varepsilon^{d-\frac{d p}{q}}\right)^{\frac{1}{p}}\|f\|_{\mathcal{M}_{q}^{p}}}{\|f\|_{\mathcal{M}_{q}^{p}}}>3(1-\delta) .
$$

This proves that $\mathcal{M}_{q}^{p}$ is not uniformly non- $\ell_{3}^{1}$.

In order to reveal the pattern, we shall now present the proof for $n=4$. With similar notations as above, we write

$$
\begin{aligned}
& f_{1}:=(+1,+1,+1,+1,+1,+1,+1,+1), \\
& f_{2}:=(+1,+1,+1,+1,-1,-1,-1,-1), \\
& f_{3}:=(+1,+1,-1,-1,+1,+1,-1,-1), \\
& f_{4}:=(+1,-1,+1,-1,+1,-1,+1,-1),
\end{aligned}
$$

where the $i$-th term corresponds to the sign of $f_{\varepsilon, 8-i}$ for $i=1,2, \ldots, 8$. Observe that

$$
\left\|f_{i}\right\|_{\mathcal{M}_{q}^{p}}=\left\|f \chi_{\left\{x: \varepsilon^{8}<|x|<1\right\}}\right\|_{\mathcal{M}_{q}^{p}}
$$

for $i=1, \ldots, 4$, and that

$$
\begin{aligned}
& 4 f_{\varepsilon, 7} \leqslant\left|f_{1}+f_{2}+f_{3}+f_{4}\right| \leqslant 4 f \chi_{\left\{x: \varepsilon^{8}<|x|<1\right\}}, \\
& 4 f_{\varepsilon, 6} \leqslant\left|f_{1}+f_{2}+f_{3}-f_{4}\right| \leqslant 4 f \chi_{\left\{x: \varepsilon^{8}<|x|<1\right\}}, \\
& 4 f_{\varepsilon, 5} \leqslant\left|f_{1}+f_{2}-f_{3}+f_{4}\right| \leqslant 4 f \chi_{\left\{x: \varepsilon^{8}<|x|<1\right\}}, \\
& 4 f_{\varepsilon, 4} \leqslant\left|f_{1}+f_{2}-f_{3}-f_{4}\right| \leqslant 4 f \chi_{\left\{x: \varepsilon^{8}<|x|<1\right\}}, \\
& 4 f_{\varepsilon, 3} \leqslant\left|f_{1}-f_{2}+f_{3}+f_{4}\right| \leqslant 4 f \chi_{\left\{x: \varepsilon^{8}<|x|<1\right\}}, \\
& 4 f_{\varepsilon, 2} \leqslant\left|f_{1}-f_{2}+f_{3}-f_{4}\right| \leqslant 4 f \chi_{\left\{x: \varepsilon^{8}<|x|<1\right\}}, \\
& 4 f_{\varepsilon, 1} \leqslant\left|f_{1}-f_{2}-f_{3}+f_{4}\right| \leqslant 4 f \chi_{\left\{x: \varepsilon^{8}<|x|<1\right\}}, \\
& 4 f_{\varepsilon, 0} \leqslant\left|f_{1}-f_{2}-f_{3}-f_{4}\right| \leqslant 4 f \chi_{\left\{x: \varepsilon^{8}<|x|<1\right\}} .
\end{aligned}
$$

Taking the Morrey norms, we get

$$
4\left(1-\varepsilon^{d-\frac{d p}{q}}\right)^{\frac{1}{p}}\|f\|_{\mathcal{M}_{q}^{p}} \leqslant\left\|f_{1} \pm f_{2} \pm f_{3} \pm f_{4}\right\|_{\mathcal{M}_{q}^{p}} \leqslant 4\left\|f \chi_{\left\{x: \varepsilon^{8}<|x|<1\right\}}\right\|_{\mathcal{M}_{q}^{p}}
$$

for all choices of signs. Taking $F_{i}:=\frac{f_{i}}{\left\|f_{i}\right\|_{\mathcal{M}_{q}^{p}}}$, we obtain $\left\|F_{i}\right\|_{\mathcal{M}_{q}^{p}}=1$ for $i=1, \ldots, 4$. By our choice of $\varepsilon$ and the fact that $\left\|f_{i}\right\|_{\mathcal{M}_{q}^{p}}=\left\|f \chi_{\left\{x: \varepsilon^{8}<|x|<1\right\}}\right\|_{\mathcal{M}_{q}^{p}}$ for $i=1, \ldots, 4$, we get

$$
\left\|F_{1} \pm F_{2} \pm F_{3} \pm F_{4}\right\|_{\mathcal{M}_{q}^{p}}>4(1-\delta) .
$$

Hence $\mathcal{M}_{q}^{p}$ is not uniformly non- $\ell_{4}^{1}$. Continuing the pattern, we see that $\mathcal{M}_{q}^{p}$ is not uniformly non- $\ell_{n}^{1}$ for $n \geqslant 2$. 


\section{3. $n$-Th Von Neumann-Jordan Constant and $n$-Th James Constant}

In this section we assume that $n \geqslant 2$. For a Banach space $\left(X,\|\cdot\|_{X}\right)$, the $n$-th Von NeumannJordan constant $C_{\mathrm{NJ}}^{(n)}(X)[9]$ and the $n$-th James constant $C_{\mathrm{J}}^{(n)}(X)$ [10] are defined by

$$
C_{\mathrm{NJ}}^{(n)}(X):=\sup \left\{\frac{\sum_{ \pm}\left\|x_{1} \pm \cdots \pm x_{n}\right\|_{X}^{2}}{2^{n-1} \sum_{i=1}^{n}\left\|x_{i}\right\|_{X}^{2}}: x_{i} \neq 0, i=1, \ldots, n\right\},
$$

and

$$
\begin{gathered}
C_{\mathrm{J}}^{(n)}(X):=\sup \left\{\min \left\{\left\|x_{1} \pm \cdots \pm x_{n}\right\|_{X}: \text { all possible choices of signs }\right\}:\right. \\
\left.x_{i} \in S_{X}, i=1, \ldots, n\right\},
\end{gathered}
$$

respectively. In the definition of $C_{\mathrm{NJ}}^{(n)}(X)$, the sum $\sum_{ \pm}$is taken over all possible choices of signs.

We state some results about the two constants. The last one is specific for Morrey spaces.

Theorem 3.1. 9] For a general Banach space $\left(X,\|\cdot\|_{X}\right)$ we have $1 \leqslant C_{\mathrm{NJ}}^{(n)}(X) \leqslant n$. In particular, $C_{\mathrm{NJ}}^{(n)}(X)=1$ if and only if $X$ is a Hilbert space.

Theorem 3.2. [10] For a Banach space $\left(X,\|\cdot\|_{X}\right)$ in general, we have $1 \leqslant C_{\mathrm{J}}^{(n)}(X) \leqslant n$. If $\operatorname{dim}(X)=\infty$, then $\sqrt{n} \leqslant C_{\mathrm{J}}^{(n)}(X) \leqslant n$. For a Hilbert space $\left(X,\langle\cdot, \cdot\rangle_{X}\right)$, we have $C_{\mathrm{J}}^{(n)}(X)=\sqrt{n}$.

Theorem 3.3. For a general Banach space $\left(X,\|\cdot\|_{X}\right)$ we have

$$
\left[C_{\mathrm{J}}^{(n)}(X)\right]^{2} \leqslant n C_{\mathrm{NJ}}^{(n)}(X) .
$$

Proof. For every $x_{i} \in S_{X}, i=1, \ldots, n$, let

$$
m:=\min \left\{\left\|x_{1} \pm \cdots \pm x_{n}\right\|_{X} \text { : all possible choices of signs }\right\} .
$$

Then, clearly $m \leqslant\left(\prod_{ \pm}\left\|x_{1} \pm \cdots \pm x_{n}\right\|_{X}\right)^{\frac{1}{n}}$, where the product is taken over all possible choices of signs. Next, by the GM-QM inequality and the last inequality, we have

$$
m \leqslant\left(\frac{\sum_{ \pm}\left\|x_{1} \pm \cdots \pm x_{n}\right\|_{X}^{2}}{2^{n-1}}\right)^{\frac{1}{2}}=\left(n \cdot \frac{\sum_{ \pm}\left\|x_{1} \pm \cdots \pm x_{n}\right\|_{X}^{2}}{2^{n-1} \sum_{i=1}^{n}\left\|x_{i}\right\|_{X}^{2}}\right)^{\frac{1}{2}} \leqslant\left(n C_{\mathrm{NJ}}^{(n)}(X)\right)^{\frac{1}{2}} .
$$

Taking the supremum over all $x_{i} \in S_{X}, i=1, \ldots, n$, the desired inequality follows.

Theorem 3.4. For $1 \leqslant p<q<\infty$, we have $C_{\mathrm{J}}^{(n)}\left(\mathcal{M}_{q}^{p}\right)=C_{\mathrm{NJ}}^{(n)}\left(\mathcal{M}_{q}^{p}\right)=n$.

Proof. It follows immediately from Theorem 2.1 that $C_{\mathrm{J}}^{(n)}\left(\mathcal{M}_{q}^{p}\right)=n$. Combining this fact and Theorem 3.3, we get $C_{\mathrm{NJ}}^{(n)}\left(\mathcal{M}_{q}^{p}\right) \geqslant n$. On the other hand, by Theorem 3.1, we have $C_{\mathrm{NJ}}^{(n)}\left(\mathcal{M}_{q}^{p}\right) \leqslant n$. Thus, $C_{\mathrm{NJ}}^{(n)}\left(\mathcal{M}_{q}^{p}\right)=n$.

\section{Concluding Remarks}

Before we end our paper, let us consider a Banach space $\left(X,\|\cdot\|_{X}\right)$ which is uniformly $n$-convex, that is, for each $\varepsilon \in(0, n)$ there exists $\delta \in(0,1)$ such that for all $x_{1}, \ldots, x_{n} \in$ $S_{X}$ with $\left\|x_{1} \pm \cdots \pm x_{n}\right\|_{X}>\varepsilon$ for all choices of signs except for $\left\|x_{1}+\cdots+x_{n}\right\|_{X}$, we have $\left\|x_{1}+\cdots+x_{n}\right\|_{X} \leqslant n(1-\delta)$. This condition is stronger than the uniformly non- $\ell_{n}^{1}$ condition, as we state in the following theorem.

Theorem 4.1. If $X$ is uniformly $n$-convex, then $X$ is uniformly non- $\ell_{n}^{1}$.

Proof. Take an $\varepsilon \in(0, n)$ and choose a corresponding $\delta \in(0,1)$ such that for all $x_{1}, \ldots, x_{n} \in S_{X}$ with $\left\|x_{1} \pm \cdots \pm x_{n}\right\|_{X}>\varepsilon$ for all choices of signs except for $\left\|x_{1}+\cdots+x_{n}\right\|_{X}$, we have $\left\|x_{1}+\cdots+x_{n}\right\|_{X} \leqslant n(1-\delta)$. Observe that if $n(1-\delta) \geqslant \varepsilon$, then we are done. Otherwise, we choose $\delta_{0} \in(0, \delta)$ such that $n\left(1-\delta_{0}\right) \geqslant \varepsilon$. This $\delta_{0}$ satisfies the uniformly non- $\ell_{n}^{1}$ condition. 
As a consequence of the above theorem and the fact that, for $1 \leqslant p<q<\infty$, the Morrey space $\mathcal{M}_{q}^{p}$ is not uniformly non- $\ell_{n}^{1}$, we conclude that $\mathcal{M}_{q}^{p}$ is not uniformly $n$-convex.

\section{ACKNOWLEDGEMENTS}

We thank H. Batkunde, Ifronika, and N.K. Tumalun for useful discussions regarding the $n$-th James constant and $n$-th Von Neumann-Jordan constant for Banach spaces.

\section{BIBLIOGRAPHY}

1. F. Chiarenza and M. Frasca. A remark on a paper by C. Fefferman // Proc. Amer. Math. Soc. 108:2, 407-409 (1990).

2. J. A. Clarkson. The von Neumann-Jordan constant for the Lebesgue spaces // Ann. Math. 38:1, 114-115 (1937).

3. J. Gao and K.-S. Lau. On the geometry of spheres in normed linear spaces // J. Austral. Math. Soc. 48:1, 101-112 (1990).

4. H. Gunawan, E. Kikianty, Y. Sawano, and C. Schwanke. Three geometric constants for Morrey spaces // Bull. Korean Math. Soc. 56:6, 1569-1575 (2019).

5. R. C. James. Uniformly non-square Banach spaces // Ann. Math. 80:3, 542-550 (1964).

6. A. Jiménez-Melado, E. Llorens-Fuster, and E. Mazcunán-Navarro. The Dunkl-Williams constant, convexity, smoothness and normal structure // J. Math. Anal. Appl. 342:1, 298-310 (2008).

7. P. Jordan and J. Von Neumann. On inner products in linear, metric spaces // Ann. Math. 36:3, 719-723 (1935).

8. M. Kato, L. Maligranda, and Y. Takahashi. On James and Jordan-von Neumann constants and the normal structure coefficient of Banach spaces // Studia Math. 144:3, 275-295 (2001).

9. M. Kato, Y. Takahashi, and K. Hashimoto. On n-th Von Neumann-Jordan constants for Banach spaces // Bull. Kyushu Inst. Tech. No. 45, 25-33 (1998).

10. L. Maligandra, L.I. Nikolova, L.-E. Persson, T. Zachariades. On n-th James and Khintchine constants of Banach spaces // Math. Ineq. Appl. 11:1, 1-22 (2007).

11. A. Muta'zili and H. Gunawan. On geometric constants for (small) Morrey spaces // Preprint: arXiv:1904.01712 (2019).

12. Y. Sawano. A thought on generalized Morrey spaces // J. Indones. Math. Soc. 25:3, 210-281 (2019).

Hendra Gunawan,

Faculty of Mathematics and Natural Sciences,

Jalan Ganesha No. 10,

Bandung,

40132, Indonesia

E-mail: hgunawan@math.itb.ac.id

Denny Ivanal Hakim,

Faculty of Mathematics and Natural Sciences,

Jalan Ganesha No. 10,

Bandung,

40132, Indonesia

E-mail: dhakim@math.itb.ac.id

Arini Soesatyo Putri,

Faculty of Mathematics and Natural Sciences, Jalan Ganesha No. 10,

Bandung,

40132, Indonesia

E-mail: arinisputri@students.itb.ac.id 